\title{
ON EXCEPTIONAL VALUES OF A MEROMORPHIC FUNCTION
}

\author{
MAKOTO OHTSUKA
}

1. M. Brelot [1] has shown that if $u(z)$ is subharmonic in an open set $D$ in the $z$-plane with boundary $C$ and is bounded from above in a neighborhood of a boundary point $z_{0}$, which is contained in a set $E \subset C$ of inner harmonic measure zero with respect to $D$, and such that $z_{0}$ is a regular point for Dirichlet problem in $D$, then

$$
\varlimsup_{\substack{z \rightarrow z_{0} \\ z \in D}} u(z)=\varlimsup_{\substack{z^{z^{\prime}} \rightarrow z_{0} \\ z^{\prime} \in C-E}}\left(\varlimsup_{\substack{z \rightarrow z^{\prime} \\ z \in D}} u(z)\right) .
$$

Furthermore, it was shown that if $f(z)$ is meromorphic in $D$, then, for any $z_{0}$ of $E$, which is in the closure of $C-E$, whether a regular point or not, the same relation holds when $u(z)$ is replaced by $|f(z)|$ whenever the left side of (1) is finite. It is easy to see that this last relation is equivalent to the relation: ${ }^{1)}$

$$
\text { boundary of } S_{z_{0}}^{(D)} \subset S_{z_{0}}^{(C-E)} \text {, }
$$

where the cluster set $S_{z_{0}}^{(D)}$ is the set of values approached sequencewise by $f(z)$ in any neighborhood of $z_{0}$ and the boundary cluster set $S_{z_{0}}^{(C-E)}$ from $C-E$ is the limit of the closure of $\underset{z^{\prime} \in(C-E)_{y}}{\bigcup} S_{z^{\prime}}^{(n)}$ as $r \rightarrow 0,(C-E)_{r}$ being that part of $C-E$ in $\left|z-z_{0}\right|<r$.

Later M. Tsuji [5] showed that in the special case that $D$ is a domain and $E$ is a closed set of logarithmic capacity zero, the exceptional values in $\Omega=S_{z_{0}}^{(D)}-S_{z_{0}}^{(C-E)}$, that is, the set of values in $\Omega$ which $f(z)$ does not assume in some neighborhood of $z_{0}$ form a set of inner logarithmic capacity zero.

2. In this note we shall prove that this is true in the general case.

Theorem. Let $D$ be an open set in the z-plane, $C$ its boundary, $E \subset C a$ set of inner harmonic measure zero with respect to $D, z_{0}$ a point of $E$ in the closure of $C-E$, and $f(z)$ a meromorphic function in $D$. Then every value of

Received May 30, 1955.

1) See [4], for instance. 
$S_{z_{0}}^{(D)}-S_{z_{0}}^{(C-E)}$ is assumed by $f(z)$ in any neighborhood of $z_{0}$ except for a set of (outer) logarithmic capacity zero.

Proof. If there is a disc: $\left|z-z_{0}\right|<r$ such that the part $C_{r}$ of $C$ in this disc is contained in $E$, this part is of harmonic measure zero with respect to this disc minus $C_{r}$ and hence of logarithmic capacity zero. Hence in any neighborhood of $z_{0}$ in $D f(z)$ takes on every complex value except for a closed set of logarithmic capacity zero, or else $f(z)$ is continuous at $z_{0}$, by a theorem of Kametani [3]. Thus our theorem is true in this case.

Next we consider the case when $z_{0}$ is in the closure of $C-E$ and suppose that the exceptional values in $S_{z_{0}}^{(D)}-S_{z_{0}}^{(C-E)}$ form a set of positive inner logarithmic capacity. Then there exists a closed bounded set $F$ of positive logarithmic capacity lying in a component $\Omega_{1}$ of $S_{z_{0}}^{(D)}-S_{z_{0}}^{(C-E)}$ such that the values of $F$ are not assumed by $f(z)$ in $D_{r_{0}}: D \cap\left\{\left|z-z_{0}\right|<r_{0}\right\}$. Let $K$ be a compact set in $\Omega_{1}$ containing a closed subset $F_{1} \subset F$ of positive logarithmic capacity in its interior and bounded by a smooth curve $\gamma$. If we take $r_{1}<r_{0}$ sufficiently small, $K$ is disjoint from the closure of $\bigcup_{z^{\prime} \in(C-E) r_{1}} S_{z^{\prime}}^{(D)}$. Brelot's result (2) shows that $S_{z^{\prime}}^{(D)} \cap K=\phi$ or $S_{z^{\prime}}^{(D)} \supset K$ at any regular point $z^{\prime} \in E_{r_{1}}: E \cap\left\{\left|z-z_{0}\right|<r_{1}\right\}$. However, the latter case cannot occur. For, if we apply Brelot's result (1) to the composed function in $D_{r_{1}}$ of $f(z)$ with the equilibrium potential of $F_{1}$, we get a contradiction. Therefore if we exclude all regular points from $E$ and denote the remaining set by $E_{1}$, that component of $S_{z_{0}}^{(D)}-S_{z_{0}}^{\left(C-E_{1}\right)}$ containing $K$ is equal to $\Omega_{1}$; that is, this set remains unchanged. Let us consider the inverse image in $D_{r_{1}}$ of the interior of $K$ and denote it by $D_{0}$. The boundary of $D_{0}$ consists of (i ) part of $\left|z-z_{0}\right|=r_{1}$, (ii) certain arcs in $D_{r_{1}}$ on which $f(z) \in \gamma$ and (iii) a closed subset $E_{2}$ of $E_{1}$ of logarithmic capacity zero. If there is no connected component of $D_{0}$ containing $z_{0}$ on its boundary, then $z_{0}$ is a regular point with respect to $D_{0}$ and the reasoning used above is applied again. If there is a domain containing $z_{0}$ on its boundary, we can apply the result of Tsuji, stated at the beginning, to obtain a contradiction to the fact that $K$ contains a closed set of exceptional values of positive logarithmic capacity. Since the set $B_{n}$ of values in $S_{z_{0}}^{(D)}-S_{z_{0}}^{(C-E)}$ not taken by $f(z)$ in $D_{1 / n}$ is a countable union of closed sets of logarithmic capacity zero, it is of (outer) logarithmic capacity zero. Hence the set of exceptional values which is equal to the union $\bigcup_{n} B_{n}$ is of (outer) logarithmic capacity zero. Thus our proof is completed. 
Finally we remark that if we use the ramified topology in $D$ (see [2] for this) and define the vanishing of harmonic measure and the cluster sets with respect to this topology, then we can extend our result to this case.

\section{BIBLIOGRAPHY}

[1] M. Brelot: Sur l'allure à la frontière des fonctions harmoniques, sousharmoniques ou holomorphes, Bull. Soc. Roy. Liège. (1939). pp. 468-477.

[2] M. Brelot: Le problème de Dirichlet ramifié, Ann. Univ. Grenoble, 22 (1946), pp. 167-200.

[3] S. Kametani: The exceptional values of functions with the set of capacity zero of essential singularities, Proc. Imp. Acad. Tokyo, 17 (1941), pp. 429-433.

[4] K. Noshiro: On the singularities of analytic functions, Jap. Journ. Math., 17 (1940), 37-96.

[5] M. Tsuji: On the cluster set of a meromorphic function, Proc. Imp. Acad. Tokyo, 19 (1943), pp. 6C-65.

Maihematical Institute, Nagoya University 